\title{
A COMPLETE SYSTEM OF THE SHAPES OF TRIANGLES
}

\author{
Vladimir Volenec, Zdenka Kolar-Begović and Ružica \\ KOLAR-ŠUPER \\ University of Zagreb and University of Osijek, Croatia
}

\begin{abstract}
In this paper we examine the shape of a triangle by means of a ternary operation which satisfies some properties. We prove that each system of the shapes of triangles can be obtained by means of the field with defined ternary operation. We give a geometric model of the shapes of triangles on the set of complex numbers which motivate us to introduce some geometric concepts. The concept of transfer is defined and some interesting properties are explored. By means of transfer the concept of a parallelogram is introduced.
\end{abstract}

\section{INTRODUCTION}

The concept of the shapes of triangles in the Euclidean plane is studied in the papers listed in the references. Two triangles are of the same shape if they are similar. More precisely, in the case of oriented triangles, the triangles are of the same shape if they are directly similar.

In [6], it is proved that each triangle with angles $A, B$ and $\mathrm{C}$ is assigned the number $\varepsilon=9-4\left(\sin ^{2} A+\sin ^{2} B+\sin ^{2} C\right)$, and the whole class of mutually similar triangles is represented by one number $\varepsilon$ such that $0 \leq \varepsilon \leq 9$. Equilateral triangles, acute triangles, right triangles, obtuse triangles and degenerate triangles are assigned the number 0 , the numbers $0<\varepsilon<1$, the number 1 , the numbers $1<\varepsilon<9$, and the number 9 , respectively. By means of the number $\varepsilon$ we "measure" the deviation of an individual class of mutually similar triangles from equilateral triangles. In [5], the class of mutually similar triangles is represented by a triangle from this class which is inscribed in the unit circle in the Gauss plane. If $z_{1}, z_{2}$ and $z_{3}$ are complex coordinates of the vertices of this triangle, then the numbers $\sigma=z_{1}+z_{2}+z_{3}$ and $R=|\sigma|^{2}$,

2010 Mathematics Subject Classification. 20N05.

Key words and phrases. Shape of a triangle, ternary operation, transfer. 
$Y=\left|\operatorname{Im}\left(\sigma^{3}\right)\right|$ are assigned to the considered class of triangles. The number $R$ and number $Y$ are called scaleneness and obtuseness of the considered class of triangles.

In [7] and [1], particular classes of mutually similar triangles, including degenerate triangles, are mapped to the particular points inside or on the border of a certain domain in the Euclidean plane and in this way special classes of triangles (for example, isosceles triangles and right triangles) are identified as subsets of this domain.

In [3] and [4], the triangle with complex coordinates $a, b$ and $c$ of its vertices in the Gauss plane is assigned the number $\Delta=\frac{c-a}{b-a}$, which is called the shape of that triangle, and in [2], the shape of the same triangle is defined as the number $\phi=\frac{1+\zeta \Delta}{\zeta+\Delta}$, where $\zeta=e^{\frac{2 \pi i}{3}}$.

In this paper, we will show that the algebraic background of the concept of the shape of triangles is one ternary operation with certain properties characterizing this concept.

\section{Definition of the COMPlete System of tRiangular Shapes}

Let $Q$ be a given set, in which two different elements are highlighted, which shall be labelled with 0 and 1 , and let the ternary operation [ ] : $Q^{3} \rightarrow Q,(a, b, c) \longmapsto[a b c]$ is given. For each $a \in Q$ the binary operation $\cdot_{a}: Q^{2} \rightarrow Q$ is defined by the formula

$$
x \cdot{ }_{a} y=[x a y] .
$$

We shall say that $(Q,[])$ is a complete system of the shapes of triangles if these seven properties are satisfied:

S1. $\left(Q, \cdot_{a}\right)$ is a quasigroup for each $a \in Q \backslash\{0,1\}$, i.e. for each $x, z \in Q$ there is a unique element $y \in Q$, and for each $y, z \in Q$ there is a unique element $x \in Q$, such that the equality $x \cdot{ }_{a} y=z$, i.e. the equality $[x a y]=z$, holds.

S2. A quasigroup $\left(Q, \cdot_{a}\right)$ is idempotent for each $a \in Q \backslash\{0,1\}$, i.e. the identity $x \cdot{ }_{a} x=x$, i.e. the identity $[x a x]=x$, holds.

S3. Quasigroups $\left(Q, \cdot_{a}\right)$ and $\left(Q, \cdot{ }^{\cdot}\right)$ are mutually medial for each $a, b \in$ $Q \backslash\{0,1\}$ i.e. the identity

$$
\left(x \cdot{ }_{a} y\right) \cdot{ }_{b}\left(u \cdot{ }_{a} v\right)=(x \cdot b u) \cdot{ }_{a}(y \cdot b v),
$$

i.e. identity

$$
[[x a y] b[u a v]]=[[x b u] a[y b v]],
$$

holds.

S4. The identity $[x 0 y]=x$, i.e. the identity $x \cdot 0 y=x$, holds.

S5. The identity $[x 1 y]=y$, i.e. the identity $x \cdot 1 y=y$, holds.

S6. The identity $[0 x y]=[0 y x]$ holds.

S7. The identity $[a[1 b 0] c]=[c b a]$ holds. 
For every $a \in Q$ the binary operation $\cdot{ }_{a}$ shall be called a triangular shape (a). S3 implies that the quasigroup $\left(Q, \cdot{ }_{a}\right)$ is medial, i.e. the identity $\left(x \cdot{ }_{a} y\right) \cdot \cdot_{a}\left(u \cdot{ }_{a} v\right)=\left(x \cdot \cdot_{a} u\right) \cdot{ }_{a}\left(y \cdot{ }_{a} v\right)$ holds.

Example 2.1. If $(F,+, \cdot)$ is a field and [ $]: F^{3} \rightarrow F$ a ternary operation defined by the formula

$$
[x a y]=(1-a) x+a y,
$$

then $(F,[])$ is a complete system of the shapes of triangles. We will show later that each system of the shapes of triangles can be obtained by means of some field in this way.

In the special case when $F=\mathbb{C}$ is the set of complex numbers, the equality $z=[x a y]$, i.e. $z=(1-a) x+a y$, can be written in the form

$$
\frac{z-x}{y-x}=a=\frac{a-0}{1-0}
$$

which means that in the Gauss plane the triangle with the vertices $x, y, z$ is directly similar to the triangle with the vertices $0,1, a$, which is degenerate in the case of a real number $a$. This model of the shapes of triangles motivated us to define this concept and introduce geometry-related terminology.

\section{TRANSFERS}

Let $(Q,[])$ be any complete system of the shapes of triangles. The elements of the set $Q$ will be called points.

For any $a \in Q \backslash\{0,1\}$ and any $p \in Q$ the bijections defined by the formulas

$$
{ }_{a} \lambda_{p}(x)=p \cdot{ }_{a} x=[p a x], \quad{ }_{a} \rho_{p}(x)=x \cdot{ }_{a} p=[x a p]
$$

will be called left transfer ${ }_{a} \lambda_{p}$ and right transfer ${ }_{a} \rho_{p}$ of a quasigroup $\left(Q, \cdot{ }_{a}\right)$, respectively.

For any $a \in Q \backslash\{0,1\}$ and any $p, q \in Q$ the bijections

$$
{ }_{a} \lambda_{p, q}={ }_{a} \lambda_{q}{ }^{-1} \circ{ }_{a} \lambda_{p}, \quad{ }_{a} \rho_{p, q}={ }_{a} \rho_{q}{ }^{-1} \circ{ }_{a} \rho_{p}
$$

will be called left and right a-transfer, respectively, where $\circ$ is the composition of mapping.

Owing to (3.1), these definitions imply the equivalencies

$$
\begin{aligned}
& { }_{a} \lambda_{p, q}(x)=y \Leftrightarrow{ }_{a} \lambda_{p}(x)={ }_{a} \lambda_{q}(y) \Leftrightarrow p \cdot{ }_{a} x=q \cdot{ }_{a} y, \\
& { }_{a} \rho_{p, q}(x)=y \Leftrightarrow{ }_{a} \rho_{p}(x)={ }_{a} \rho_{q}(y) \Leftrightarrow x \cdot{ }_{a} p=y \cdot{ }_{a} q .
\end{aligned}
$$

The equation $p \cdot{ }_{a} x=q \cdot{ }_{a} y$, i.e. $x \cdot{ }_{a} p=y \cdot{ }_{a} q$, has a unique solution $p$ or $q$ if three other elements are given. Because of that, we have the following statement.

THEOREM 3.1. If the points $p, x, y$ are given, then there is a unique point $q$, and if the points $q, x, y$ are given, then there is a unique point $p$ such that the equality ${ }_{a} \lambda_{p, q}(x)=y$, i.e. ${ }_{a} \rho_{p, q}(x)=y$, holds. 
Theorem 3.2. Let ${ }_{a} \lambda_{p, q}={ }_{a} \lambda_{r, s}$ or ${ }_{a} \rho_{p, q}={ }_{a} \rho_{r, s}$. The equalities $p=r$ and $q=s$ are equivalent.

Proof. Let ${ }_{a} \lambda_{p, q}={ }_{a} \lambda_{r, s}$, let $x$ be a given point and let

$$
y={ }_{a} \lambda_{p, q}(x)={ }_{a} \lambda_{r, s}(x) .
$$

Due to (3.2), we get equalities $p \cdot{ }_{a} x=q \cdot_{a} y, r \cdot{ }_{a} x=s \cdot{ }_{a} y$. If $p=r$, then we get $q \cdot{ }_{a} y=s \cdot{ }_{a} y$, from where, because of S1, it follows that $q=s$, and if $q=s$, then $p \cdot \cdot_{a} x=r \cdot{ }_{a} x$, from where we obtain $p=r$. The equality ${ }_{a} \rho_{p, q}={ }_{a} \rho_{r, s}$ can be studied similarly.

Theorem 3.3. Let $a, b \in Q \backslash\{0,1\}$. If ${ }_{a} \lambda_{p, q}(u)={ }_{b} \rho_{r, s}(u)$ for some point $u$, then ${ }_{b} \lambda_{p, q}={ }_{a} \rho_{r, s}$.

Proof. Let $v={ }_{a} \lambda_{p, q}(u)={ }_{b} \rho_{r, s}(u)$. If $x$ is any point, then let $y=$ ${ }_{b} \lambda_{p, q}(x)$. Owing to (3.2) and (3.3), we get equalities $p \cdot{ }_{a} u=q \cdot{ }_{a} v, p \cdot{ }_{b} x=q \cdot{ }_{b} y$, $u \cdot{ }_{b} r=v \cdot b s$. Now property S3 implies

$$
\begin{aligned}
\left(p \cdot{ }_{a} u\right) \cdot{ }_{b}\left(x \cdot{ }_{a} r\right) & =\left(p \cdot{ }_{b} x\right) \cdot{ }_{a}\left(u \cdot{ }_{b} r\right)=\left(q \cdot{ }_{b} y\right) \cdot{ }_{a}\left(v \cdot{ }_{b} s\right) \\
& =\left(q \cdot{ }_{a} v\right) \cdot{ }_{b}\left(y \cdot{ }_{a} s\right)=\left(p \cdot \cdot_{a} u\right) \cdot{ }_{b}\left(y \cdot{ }_{a} s\right),
\end{aligned}
$$

from where by cancelation in a quasigroup $(Q, \cdot b)$ (property S1) it follows $x \cdot{ }_{a} r=y \cdot{ }_{a} s$, based upon (3.3), it means that $y={ }_{a} \rho_{r, s}(x)$ and then ${ }_{b} \lambda_{p, q}(x)=$ ${ }_{a} \rho_{r, s}(x)$ for each point $x$. Therefore ${ }_{b} \lambda_{p, q}={ }_{a} \rho_{r, s}$.

Corollary 3.4. Let $a \in Q \backslash\{0,1\}$. If ${ }_{a} \lambda_{p, q}(u)={ }_{a} \rho_{r, s}(u)$ for some point $u$, then ${ }_{a} \lambda_{p, q}={ }_{a} \rho_{r, s}$.

TheOREM 3.5. Let $a, b \in Q \backslash\{0,1\}$ and if any three of four points $p, q, r$ and $s$ are given, then the remaining point of these points is uniquely determined such that ${ }_{b} \lambda_{p, q}={ }_{a} \rho_{r, s}$.

Proof. Let the points $p, q$ be given. Let the point $u$ be given and let $v={ }_{a} \lambda_{p, q}(u)$. According to Theorem 3.1, there is a point $s$ if the point $r$ is given and there is a point $r$ if the point $s$ is given such that ${ }_{b} \rho_{r, s}(u)=v$ in both cases. Therefore, we have ${ }_{a} \lambda_{p, q}(u)={ }_{b} \rho_{r, s}(u)$, then Theorem 3.3 implies ${ }_{b} \lambda_{p, q}={ }_{a} \rho_{r, s}$. Now, let ${ }_{b} \lambda_{p, q}={ }_{a} \rho_{r, s^{\prime}}$ or ${ }_{b} \lambda_{p, q}={ }_{a} \rho_{r^{\prime}, s}$. Then we get ${ }_{a} \rho_{r, s}={ }_{a} \rho_{r, s^{\prime}}$, i.e. ${ }_{a} \rho_{r, s}={ }_{a} \rho_{r^{\prime}, s}$, and by Theorem 3.2 there follows $s^{\prime}=s$, i.e. $r^{\prime}=r$. Analogously, it can be proved that with the given points $p, r, s$ and $q, r, s$, the point $q$ and the point $p$ are uniquely determined, respectively, so that ${ }_{b} \lambda_{p, q}={ }_{a} \rho_{r, s}$.

TheOrem 3.6. Let $a \in Q \backslash\{0,1\}$. If ${ }_{a} \lambda_{p, q}(u)={ }_{a} \lambda_{r, s}(u)$ for any point $u$, then ${ }_{a} \lambda_{p, q}={ }_{a} \lambda_{r, s}$. If ${ }_{a} \rho_{p, q}(u)={ }_{a} \rho_{r, s}(u)$ for any point $u$, then ${ }_{a} \rho_{p, q}={ }_{a} \rho_{r, s}$.

Proof. Suppose $v={ }_{a} \lambda_{p, q}(u)={ }_{a} \lambda_{r, s}(u)$ and let $e$ be a given point. According to Theorem 3.1, there is a point $f$ such that ${ }_{a} \rho_{e, f}(u)=v$. From ${ }_{a} \lambda_{p, q}(u)={ }_{a} \rho_{e, f}(u)$ and Corollary 3.4 there follows ${ }_{a} \lambda_{p, q}={ }_{a} \rho_{e, f}$. Now 
${ }_{a} \lambda_{r, s}(u)={ }_{a} \rho_{e, f}(u)$ and the same corollary imply ${ }_{a} \lambda_{r, s}={ }_{a} \rho_{e, f}$. Hence ${ }_{a} \lambda_{p, q}={ }_{a} \lambda_{r, s}$. The second statement may be proved analogously.

TheOREM 3.7. Let $a \in Q \backslash\{0,1\}$. For any three of the points $p, q, r$ and $s$ there is a unique fourth point such that ${ }_{a} \lambda_{p, q}={ }_{a} \lambda_{r, s}$. For any three of the points $p, q, r$ and $s$ there is a unique fourth point such that ${ }_{a} \rho_{p, q}={ }_{a} \rho_{r, s}$.

Proof. Let the points $p, q$ be given. Let $u$ be a given point and suppose that $v={ }_{a} \lambda_{p, q}(u)$. If the point $r$ is given, then according to Theorem 3.1, there is a point $s$, and if the point $s$ is given, then there is a point $r$ such that the equality ${ }_{a} \lambda_{r, s}(u)=v$ holds in both cases. Therefore we have ${ }_{a} \lambda_{p, q}(u)=$ ${ }_{a} \lambda_{r, s}(u)$ and then ${ }_{a} \lambda_{p, q}={ }_{a} \lambda_{r, s}$ follows from Theorem 3.6. Suppose that ${ }_{a} \lambda_{p, q}={ }_{a} \lambda_{r, s^{\prime}}$ or ${ }_{a} \lambda_{p, q}={ }_{a} \lambda_{r^{\prime}, s}$. Then we get ${ }_{a} \lambda_{r, s}={ }_{a} \lambda_{r, s^{\prime}}$, i.e. ${ }_{a} \lambda_{r, s}={ }_{a} \lambda_{r^{\prime}, s}$. Then by Theorem 3.2 there follows $s^{\prime}=s$, i.e. $r^{\prime}=r$. Analogously, it can be proved that with the given points $p, r, s$ and $q, r, s$, we uniquely determine the points $q$ and $p$, respectively, such that ${ }_{a} \lambda_{p, q}={ }_{a} \lambda_{r, s}$. Similarly, we can prove the second statement of the theorem.

If $a, b \in Q \backslash\{0,1\}$ are any points, then by Theorem 3.5 each right $a-$ transfer is also left $b$-transfer, and each left $b$-transfer is also right $a$-transfer. Thus for each $a \in Q \backslash\{0,1\}$ all left and right $a$-transfers are simply called transfers.

THEOREM 3.8. The equalities ${ }_{a} \lambda_{p, q}(r)=s$ and ${ }_{a} \rho_{r, s}(p)=q$ are equivalent.

Proof. By (3.2) and (3.3), both equalities are equivalent to $p \cdot{ }_{a} r=q \cdot{ }_{a} s$.

THEOREM 3.9. The equalities ${ }_{a} \lambda_{p, q}={ }_{a} \lambda_{r, s}$ and ${ }_{a} \rho_{p, q}={ }_{a} \rho_{r, s}$ are equivalent.

Proof. Suppose that ${ }_{a} \lambda_{p, q}={ }_{a} \lambda_{r, s}$. Let $e, g$ be any points and $f=$ ${ }_{a} \lambda_{p, q}(e)={ }_{a} \lambda_{r, s}(e), h={ }_{a} \rho_{p, q}(g)$. By Theorem 3.8, these equalities imply the equalities ${ }_{a} \rho_{e, f}(p)=q,{ }_{a} \rho_{e, f}(r)=s,{ }_{a} \lambda_{g, h}(p)=q$. The third and the first of these equalities imply by Theorem 3.3 the equality ${ }_{a} \lambda_{g, h}={ }_{a} \rho_{e, f}$, and the second equality implies ${ }_{a} \lambda_{g, h}(r)=s$. Finally, by Theorem 3.8, we have ${ }_{a} \rho_{r, s}(g)=h$, which together with $h={ }_{a} \rho_{p, q}(g)$, by Theorem 3.6, implies ${ }_{a} \rho_{p, q}={ }_{a} \rho_{r, s}$. The converse can be proved analogously.

TheOREM 3.10. The equalities ${ }_{a} \lambda_{p, q}={ }_{a} \lambda_{r, s},{ }_{a} \rho_{p, q}={ }_{a} \rho_{r, s},{ }_{a} \lambda_{p, r}={ }_{a} \lambda_{q, s}$, ${ }_{a} \rho_{p, r}={ }_{a} \rho_{q, s}$ are equivalent.

ProOF. First we notice from Theorem 3.9 that it is sufficient to prove the equivalence of the first and the fourth equality. Suppose that the first equality is true. Let $e, g$ be given points and $f={ }_{a} \lambda_{p, q}(e)={ }_{a} \lambda_{r, s}(e), h={ }_{a} \rho_{p, r}(g)$. By 
(3.2) and (3.3) these equalities imply $p \cdot{ }_{a} e=q \cdot{ }_{a} f, r \cdot{ }_{a} e=s \cdot{ }_{a} f, g \cdot{ }_{a} p=h \cdot{ }_{a} r$, and by property $\mathrm{S} 3$ therefrom follows

$$
\begin{aligned}
\left(g \cdot{ }_{a} q\right) \cdot{ }_{a}\left(r \cdot{ }_{a} f\right) & =\left(g \cdot a_{a} r\right) \cdot{ }_{a}\left(q \cdot{ }_{a} f\right)=\left(g \cdot{ }_{a} r\right) \cdot{ }_{a}\left(p \cdot{ }_{a} e\right)=\left(g \cdot{ }_{a} p\right) \cdot{ }_{a}\left(r \cdot{ }_{a} e\right) \\
& =\left(h \cdot{ }_{a} r\right) \cdot{ }_{a}\left(s \cdot{ }_{a} f\right)=(h \cdot a s) \cdot{ }_{a}\left(r \cdot{ }_{a} f\right) .
\end{aligned}
$$

Thus by (3.3) we obtain $g \cdot{ }_{a} q=h \cdot_{a} s$, i.e. ${ }_{a} \rho_{q, s}(g)=h$, which together with $h={ }_{a} \rho_{p, r}(g)$ implies ${ }_{a} \rho_{p, r}={ }_{a} \rho_{q, s}$ by Theorem 3.6. The converse can be proved analogously.

THEOREM 3.11. Let $T$ be a set of all transfers. Then $(T, \circ)$ is a commutative group, which acts strictly transitively on the set $Q$.

Proof. Let $\tau_{1}, \tau_{2}$ be any transfers and $p$ a point. By Theorems 3.5 or 3.7 there are points $q, r$ such that $\tau_{1}={ }_{a} \lambda_{p, q}, \tau_{2}={ }_{a} \lambda_{p, r}$ and the point $s$ such that $\tau_{1}={ }_{a} \lambda_{r, s}$. Therefore ${ }_{a} \lambda_{p, q}={ }_{a} \lambda_{r, s}$, and by Theorem 3.10 it follows ${ }_{a} \lambda_{p, r}={ }_{a} \lambda_{q, s}$ and then $\tau_{2}={ }_{a} \lambda_{q, s}$. Now we have

$$
\tau_{2} \circ \tau_{1}={ }_{a} \lambda_{q, s} \circ{ }_{a} \lambda_{p, q}={ }_{a} \lambda_{s}{ }^{-1} \circ{ }_{a} \lambda_{q} \circ{ }_{a} \lambda_{q}{ }^{-1} \circ{ }_{a} \lambda_{p}={ }_{a} \lambda_{s}{ }^{-1} \circ{ }_{a} \lambda_{p}={ }_{a} \lambda_{p, s},
$$

so $\tau_{2} \circ \tau_{1}$ is a transfer. On the other hand, we get analogously

$$
\tau_{1} \circ \tau_{2}={ }_{a} \lambda_{r, s} \circ{ }_{a} \lambda_{p, r}={ }_{a} \lambda_{p, s}=\tau_{2} \circ \tau_{1} .
$$

${ }_{a} \lambda_{p, p}$ is the identity for any point $p$. For any two points $p, q$ the inverse of a transfer ${ }_{a} \lambda_{p, q}$ is a transfer ${ }_{a} \lambda_{q, p}$ because of

$$
{ }_{a} \lambda_{q, p} \circ{ }_{a} \lambda_{p, q}={ }_{a} \lambda_{p, p} .
$$

For any two points $u, v$ there is a unique transfer $\tau$ such that $\tau(u)=v$. Indeed, by Theorem 3.1 for a given point $p$ there is a unique point $q$ such that ${ }_{a} \lambda_{p, q}(u)=v$. If $\tau$ is any transfer such that $\tau(u)=v$, then by Theorems 3.3 or 3.6 there follows $\tau={ }_{a} \lambda_{p, q}$.

Corollary 3.12. Let $a \in Q \backslash\{0,1\}$ be a given point. For every point $p, q$ and $r$ the following equalities hold:

$$
\begin{aligned}
& { }_{a} \lambda_{p, q} \circ{ }_{a} \lambda_{q, r}={ }_{a} \lambda_{q, r} \circ{ }_{a} \lambda_{p, q}={ }_{a} \lambda_{p, r}, \\
& { }_{a} \rho_{p, q} \circ{ }_{a} \rho_{q, r}={ }_{a} \rho_{q, r} \circ{ }_{a} \rho_{p, q}={ }_{a} \rho_{p, r} .
\end{aligned}
$$

Corollary 3.13. For every point $p, q$ the following equalities hold:

$$
{ }_{a} \lambda_{p, q}{ }^{-1}={ }_{a} \lambda_{q, p}, \quad{ }_{a} \rho_{p, q}{ }^{-1}={ }_{a} \rho_{q, p} .
$$

THEOREM 3.14. Any two of the three equalities

$$
{ }_{a} \lambda_{p, r}(e)=f, \quad{ }_{a} \lambda_{q, s}(g)=h, \quad{ }_{a} \lambda_{p \cdot{ }_{a} q, r \cdot{ }_{a} s}\left(e \cdot{ }_{a} g\right)=f \cdot{ }_{a} h
$$

imply the remaining one. An analogous statement holds for the right instead of left transfers. 
Proof. Because of (3.2), the equalities are equivalent successively to the equalities

$$
p \cdot{ }_{a} e=r \cdot{ }_{a} f, \quad q \cdot{ }_{a} g=s \cdot{ }_{a} h, \quad\left(p \cdot{ }_{a} q\right) \cdot{ }_{a}\left(e \cdot{ }_{a} g\right)=\left(r \cdot{ }_{a} s\right) \cdot{ }_{a}\left(f \cdot{ }_{a} h\right) .
$$

By property $\mathrm{S} 3$ the last equality is equivalent to $\left(p \cdot{ }_{a} e\right) \cdot{ }_{a}\left(q \cdot{ }_{a} g\right)=\left(r \cdot{ }_{a} f\right) \cdot{ }_{a}\left(s \cdot{ }_{a} h\right)$ and the statement of the theorem is now obvious because of property S1.

Theorem 3.15. Any two of the three equalities

$$
\begin{aligned}
{ }_{t} \lambda_{a, c} & ={ }_{t} \lambda_{e, g}, \\
{ }_{t} \lambda_{b, d} & ={ }_{t} \lambda_{f, h}, \\
{ }_{t} \lambda_{a \cdot{ }_{t} b, c \cdot{ }_{t} d} & ={ }_{t} \lambda_{e \cdot{ }_{t} f, g \cdot{ }_{t} h}
\end{aligned}
$$

imply the remaining one. An analogous statement holds for the right instead of left transfers.

Proof. Suppose that (3.4) and (3.5) hold and let $p$, and $r$ be given points. Set $q={ }_{t} \lambda_{a, c}(p)={ }_{t} \lambda_{e, g}(p), s={ }_{t} \lambda_{b, d}(r)={ }_{t} \lambda_{f, h}(r)$. According to ${ }_{t} \lambda_{a, c}(p)=q,{ }_{t} \lambda_{b, d}(r)=s$, namely ${ }_{t} \lambda_{e, g}(p)=q,{ }_{t} \lambda_{f, h}(r)=s$, by Theorem 3.14 we obtain ${ }_{t} \lambda_{a \cdot{ }_{t} b, c \cdot{ }_{t} d}\left(p \cdot{ }_{t} r\right)=q \cdot{ }_{t} s$, i.e. ${ }_{t} \lambda_{e \cdot{ }_{t} f, g \cdot{ }_{t} h}\left(p \cdot{ }_{t} r\right)=q \cdot{ }_{t} s$. However, by Theorem 3.6, the equality ${ }_{t} \lambda_{a \cdot{ } b, c_{\cdot} d}\left(p \cdot{ }_{t} r\right)={ }_{t} \lambda_{e \cdot{ }_{t} f, g \cdot{ }_{t} h}\left(p \cdot{ }_{t} r\right)$ implies equality (3.6). Now suppose that (3.4) and (3.6) hold and let $p, u$ be given points. Set $q={ }_{t} \lambda_{a, c}(p)={ }_{t} \lambda_{e, g}(p), v={ }_{t} \lambda_{a \cdot{ }_{t} b, c \cdot t}(u)={ }_{t} \lambda_{e \cdot{ }_{t} f, g \cdot{ }_{t} h}(u)$. Then there exist the points $r, s$ such that $p \cdot{ }_{t} r=u, q \cdot{ }_{t} s=v$. By Theorem 3.14, the equalities ${ }_{t} \lambda_{a, c}(p)=q,{ }_{t} \lambda_{a \cdot{ }_{t} b, c \cdot d}\left(p \cdot{ }_{t} r\right)=q \cdot{ }_{t} s$ and ${ }_{t} \lambda_{e, g}(p)=q,{ }_{t} \lambda_{e \cdot{ }_{t} f, g \cdot{ }_{t} h}\left(p \cdot{ }_{t} r\right)=q \cdot{ }_{t} s$ imply that ${ }_{t} \lambda_{b, d}(r)=s$ and ${ }_{t} \lambda_{f, h}(r)=s$, respectively. However, by Theorem 3.6, the equality ${ }_{t} \lambda_{b, d}(r)={ }_{t} \lambda_{f, h}(r)$ implies equality (3.5). Implication (3.5) $\&(3.6) \Rightarrow(3.4)$ can be proved analogously.

\section{Parallelograms}

We say that the points $a, b, c, d$ form a parallelogram and write $\operatorname{Par}(a, b$, $c, d)$ if there exists a transfer, which maps the points $a$ and $d$ successively to the points $b$ and $c$. If by $\tau_{a, b}$ we denote a transfer mapping the point $a$ to the point $b$, then the equivalence

$$
\operatorname{Par}(a, b, c, d) \Leftrightarrow \tau_{a, b}(d)=c
$$

holds.

Theorem 4.1. $\operatorname{Par}(a, b, c, d)$ holds if and only if ${ }_{t} \lambda_{a, b}={ }_{t} \lambda_{d, c}$ for some point $t$.

Proof. By Theorem 3.3 each transfer is a right $t$-transfer. Then $\operatorname{Par}(a, b, c, d)$ is equivalent to the fact that there exist the points $p, q$ such that ${ }_{t} \rho_{p, q}(a)=b,{ }_{t} \rho_{p, q}(d)=c$, i.e. by (3.3) it follows $a{ }_{t} p=b{ }_{t} q, d{ }{ }_{t} p=c \cdot{ }_{t} q$, i.e. by (3.2) it follows ${ }_{t} \lambda_{a, b}(p)=q,{ }_{t} \lambda_{d, c}(p)=q$, which is by Theorem 3.6 equivalent to ${ }_{t} \lambda_{a, b}={ }_{t} \lambda_{d, c}$. 
Corollary 4.2. If the point $t$ is given, the statement Par $(a, b, c, d)$ holds if and only if there exist the points $p, q$ such that

$$
a \cdot{ }_{t} p=b \cdot{ }_{t} q, \quad d \cdot{ }_{t} p=c \cdot{ }_{t} q .
$$

Corollary 4.3. If the statement Par $(a, b, c, d)$ holds, then the equalities $a \cdot{ }_{t} p=b \cdot{ }_{t} q, d \cdot{ }_{t} p=c \cdot{ }_{t} q$ are equivalent.

Corollary 4.4. Let $\operatorname{Par}(a, b, c, d)$. If one of the points $p, q$ satisfying $a \cdot{ }_{t} p=b{ }_{t} q, d{ }_{t} p=c \cdot{ }_{t} q$ is given, then the second one is uniquely determined.

Theorems analogous to Theorem 4.1 and Corollaries 4.2-4.4 are the following four statements.

TheOREM 4.5. Par $(a, b, c, d)$ holds if and only if ${ }_{t} \rho_{a, b}={ }_{t} \rho_{d, c}$ for some point $t$.

Corollary 4.6. With the given point the statement Par $(a, b, c, d)$ holds if and only if there exist the points $p, q$ such that

$$
p \cdot{ }_{t} a=q \cdot{ }_{t} b, \quad p \cdot{ }_{t} d=q \cdot{ }_{t} c .
$$

Corollary 4.7. Let $\operatorname{Par}(a, b, c, d)$. The equalities $p \cdot{ }_{t} a=q \cdot{ }_{t} b, p \cdot{ }_{t} d=q \cdot{ }_{t} c$ are equivalent.

Corollary 4.8. Let Par $(a, b, c, d)$. If one of the points $p, q$, such that $p \cdot{ }_{t} a=q \cdot{ }_{t} b, p \cdot{ }_{t} d=q \cdot{ }_{t} c$, is given, then the second one is uniquely determined.

TheOREM 4.9. The structure $(Q, P a r)$ is the parallelogram space, i.e. the following properties are satisfied:

P1. For any three points $a, b$ and $c$ there is a unique point $d$ such that $\operatorname{Par}(a, b, c, d)$.

P2. Par $(a, b, c, d)$ implies Par $(e, f, g, h)$, where $(e, f, g, h)$ is any cyclic permutation of $(a, b, c, d)$ or $(d, c, b, a)$.

P3. Par $(a, b, c, d)$ and Par $(c, d, e, f)$ imply $\operatorname{Par}(a, b, f, e)$.

Proof. Property P1 is an immediate consequence of Theorems 4.1 and 3.7. Suppose Par $(a, b, c, d)$ holds. Then, by Theorem $4.1,{ }_{t} \lambda_{a, b}={ }_{t} \lambda_{d, c}$ holds for some point $t$. To prove property P2 it is sufficient to prove $\operatorname{Par}(d, c, b, a)$ and $\operatorname{Par}(b, c, d, a)$, i.e. the equalities ${ }_{t} \lambda_{d, c}={ }_{t} \lambda_{a, b}$ and ${ }_{t} \lambda_{b, c}={ }_{t} \lambda_{a, d}$. However, by Theorem 3.10, ${ }_{t} \lambda_{a, b}={ }_{t} \lambda_{d, c}$ implies ${ }_{t} \lambda_{a, d}={ }_{t} \lambda_{b, c}$. Let us prove now property P3. By Theorem 4.1 $\operatorname{Par}(a, b, c, d)$ and $\operatorname{Par}(c, d, e, f)$ imply ${ }_{t} \lambda_{a, b}=$ ${ }_{t} \lambda_{d, c}$ and ${ }_{t} \lambda_{c, d}={ }_{t} \lambda_{f, e}$, i.e. by Corollary $3.13{ }_{t} \lambda_{d, c}={ }_{t} \lambda_{e, f}$. Therefore we have ${ }_{t} \lambda_{a, b}={ }_{t} \lambda_{e, f}$, i.e. by Theorem 4.1, the statement $\operatorname{Par}(a, b, f, e)$.

TheOREM 4.10. For any point $a, b$ and $c \operatorname{Par}(a, a, b, c)$ holds if and only if $b=c$.

PRoOF. It is the consequence of the equivalence ${ }_{t} \lambda_{a, a}={ }_{t} \lambda_{c, b} \Leftrightarrow b=c$ from Theorem 3.2. 
The next result immediately follows from property $\mathrm{P} 2$.

Corollary 4.11. $\operatorname{Par}(a, a, b, b)$ and $\operatorname{Par}(a, b, b, a)$ hold for any two points $a, b$.

Theorem 4.12. $\operatorname{Par}(a, b, d, e)$ and Par $(b, c, e, f)$ imply $\operatorname{Par}(c, d, f, a)$.

Proof. By P1 there exists a point $g$ such that $\operatorname{Par}(a, b, c, g)$. By P3 we obtain now the implications

$$
\begin{aligned}
& \operatorname{Par}(c, g, a, b) \& \operatorname{Par}(a, b, d, e) \Rightarrow \operatorname{Par}(c, g, e, d), \\
& \operatorname{Par}(g, a, b, c) \& \operatorname{Par}(b, c, e, f) \Rightarrow \operatorname{Par}(g, a, f, e), \\
& \operatorname{Par}(c, d, e, g) \& \operatorname{Par}(e, g, a, f) \Rightarrow \operatorname{Par}(c, d, f, a) .
\end{aligned}
$$

We have used P2 here several times.

Theorem 4.13. Any two of three statements Par $(a, b, c, d), \operatorname{Par}(e, f, g, h)$ and $\operatorname{Par}\left(a \cdot{ }_{t} e, b \cdot{ }_{t} f, c \cdot{ }_{t} g, d \cdot{ }_{t} h\right)$ imply the remaining statement for any point $t$.

Proof. The statement immediately follows from Theorem 3.15.

By (3.1) from Theorem 4.13 we get the following statement.

Corollary 4.14. Par $(a, b, c, d)$ and Par $(e, f, g, h)$ imply

$$
\operatorname{Par}([a t e],[b t f],[c t g],[d t h])
$$

for any point $t$.

\section{AdDition AND multiplication OF POINTS}

Let 0 and 1 be the points (from Introduction) satisfying S4 - S7. For any two points $a, b$ we will define sum $a+b$ by the equivalence

$$
c=a+b \Leftrightarrow \operatorname{Par}(0, a, c, b) .
$$

It means that $\operatorname{Par}(0, a, a+b, b)$ holds for any two points $a, b$.

Let us consider the function $\varphi$ mapping the set of all transfers $T$ into the set of all points $Q$ defined by $\varphi\left({ }_{t} \lambda_{0, a}\right)=a$ for some point $t$. By Theorems 3.5 and 3.7 for each transfer $\tau$ there is a unique point $a$ such that $\tau={ }_{t} \lambda_{0, a}$ and hence $\varphi$ is a bijection. Now let ${ }_{t} \lambda_{0, a}$ and ${ }_{t} \lambda_{0, b}$ be any two transfers. As $\operatorname{Par}(0, a, a+b, b)$, by Theorem 4.1 it follows ${ }_{t} \lambda_{0, a}={ }_{t} \lambda_{b, a+b}$. According to Corollary 3.12 , we get successively

$\varphi\left({ }_{t} \lambda_{0, a} \circ{ }_{t} \lambda_{0, b}\right)=\varphi\left({ }_{t} \lambda_{b, a+b} \circ{ }_{t} \lambda_{0, b}\right)=\varphi\left({ }_{t} \lambda_{0, a+b}\right)=a+b=\varphi\left({ }_{t} \lambda_{0, a}\right)+\varphi\left({ }_{t} \lambda_{0, b}\right)$,

so $\varphi$ is an isomorphism of groupoids $(T, \circ)$ and $(Q,+)$. Then by Theorem 3.11 we obtain the following statement.

TheOrem 5.1. $(Q,+)$ is a commutative group with the neutral element 0 , which is isomorphic to group $(T, \circ)$. 
TheOREM 5.2. The statements $\operatorname{Par}(a, b, c, d)$ and $a+c=b+d$ are equivalent.

Proof. From $\operatorname{Par}(0, a, a+c, c)$ and $\operatorname{Par}(a, b, c, d)$ by Theorem 4.12 it follows $\operatorname{Par}(b, a+c, d, 0)$, i.e., $\operatorname{Par}(b, 0, d, a+c)$ by P2. However, from $\operatorname{Par}(0, b, b+d, d)$, by $\mathrm{P} 2$ we get $\operatorname{Par}(b, 0, d, b+d)$ and by $\mathrm{P} 1$ we get $a+c=b+d$. Conversely, if $a+c=b+d$, then $\operatorname{Par}(0, a, a+c, c)$ and $\operatorname{Par}(0, b, b+d, d)$ can be written in the form $\operatorname{Par}(a, 0, c, a+c)$ (by P2) and $\operatorname{Par}(0, b, a+c, d)$, which by Theorem 4.12 implies $\operatorname{Par}(b, c, d, a)$, i.e. $\operatorname{Par}(a, b, c, d)$ by P2.

On the set $Q$ we are going to define multiplication - by the formula

$$
a \cdot b=[0 a b] .
$$

THEOREM 5.3. ( $Q \backslash\{0\}, \cdot)$ is a commutative group with the neutral element 1 and for each point a there holds $a \cdot 0=0$.

PROOF.

$$
a \cdot(b \cdot c)=b \cdot(a \cdot c)
$$

holds for each $a, b$ and $c \in Q$. Indeed, we get successively

$a \cdot(b \cdot c) \stackrel{(5.2)}{=}[0 a[0 b c]] \stackrel{S 2}{=}[[0 b 0] a[0 b c]] \stackrel{S 3}{=}[[0 a 0] b[0 a c]] \stackrel{S 2}{=}[0 b[0 a c]] \stackrel{(5.2)}{=} b \cdot(a \cdot c)$.

The equality

$$
a \cdot b=b \cdot a
$$

holds by S6 for each $a, b \in Q$. Now, for each $a, b, c \in Q$

$$
(a \cdot b) \cdot c \stackrel{(5.4)}{=} c \cdot(a \cdot b) \stackrel{(5.3)}{=} a \cdot(c \cdot b) \stackrel{(5.4)}{=} a \cdot(b \cdot c)
$$

holds. For each $a \in Q$ we have

$$
1 \cdot a \stackrel{(5.2)}{=}[01 a] \stackrel{S 5}{=} a .
$$

For each $a \in Q \backslash\{0,1\}$, by S1 there exists a point denoted by $a^{-1}$, such that $\left[0 a a^{-1}\right]=1$, i.e. by $(5.2) a \cdot a^{-1}=1$. Moreover, $1^{-1}=1$ since $1 \cdot 1=1$. For each $a \in Q$

$$
a \cdot 0 \stackrel{(5.2)}{=}[0 a 0] \stackrel{S 2}{=} 0
$$

holds.

TheOrem 5.4. $(Q,+, \cdot)$ is a field with the additive identity 0 and the multiplicative identity 1.

Proof. By Theorems 5.1 and 5.3 it is sufficient to prove that for each $a, b$ and $c \in Q$ it holds

$$
a \cdot(b+c)=a \cdot b+a \cdot c .
$$

However, as $a \in Q \backslash\{0,1\}$, then $\operatorname{Par}(0,0,0,0)$, which is true by Corollary 4.11, and $\operatorname{Par}(0, b, b+c, c)$ by Corollary 4.14 imply $\operatorname{Par}([0 a 0],[0 a b],[0 a(b+c)],[0 a c])$, 
i.e. by $\mathrm{S} 2$ and (5.2) $\operatorname{Par}(0, a \cdot b, a \cdot(b+c), a \cdot c)$, which by (5.1) imply (5.5). For $a=0$ or $a=1$ equality (5.5) is obvious.

THEOREM 5.5. The formula

$$
[a b c]=(1-b) a+b \cdot c
$$

holds for all points $a, b$ and $c$.

Proof. First, let $b \in Q \backslash\{0,1\}$. By Corollary 4.11 we have $\operatorname{Par}(0,1,1,0)$ and $\operatorname{Par}(0,0,1,1)$, which by Corollary 4.14 imply $\operatorname{Par}([0 b 0],[1 b 0],[1 b 1],[0 b 1])$, and by $\mathrm{S} 2$ it follows (5.2) $\operatorname{Par}(0,[1 b 0], 1, b)$. Then by (5.1) it holds $[1 b 0]+b=1$, namely the equality

$$
[1 b 0]=1-b .
$$

By Corollary 4.11 there hold $\operatorname{Par}(0, a, a, 0)$ and $\operatorname{Par}(0,0, c, c)$. By Corollary 4.14 , these imply $\operatorname{Par}([0 b 0],[a b 0],[a b c],[0 b c])$, namely by S2 and S7 $\operatorname{Par}(0,[0[1 b 0] a],[a b c],[0 b c])$, which, by (5.1) and (5.2), can be written in the form

$$
[a b c]=[1 b 0] \cdot a+b \cdot c,
$$

and by (5.7) in the form (5.6). For $b=1$ or $b=0$ equality (5.6) is obvious since by $\mathrm{S} 5$ we have $[a 1 c]=c$, and by $\mathrm{S} 4$ we get $[a 0 c]=a$.

From Theorem 5.5 it follows that each system of triangle shapes can be obtained from some field in the manner described in the example.

\section{REFERENCES}

[1] H. B. Griffiths, Triangles of triangles, Math. Gaz. 65 (1981), 10-19.

[2] M. Hajja, H. Martini and M. Spirova, On converses of Napoleon's theorem and a modified shape function, Beiträge Algebra Geom. 47 (2006), 363-383.

[3] J. A. Lester, Triangles. I. Shapes, Aequationes Math. 52 (1996), 30-54.

[4] J. A. Lester, Triangles. II. Complex triangle coordinates, Aequationes Math. 52 (1996), 215-245.

[5] D. N. Mackenzie, What is the shape of a triangle? Note Mat. 13 (1993), 237-250.

[6] A.Pařízek, Vyznačení tvaru trojúhelniku veličinou, Čas. Pěst. Math. Fys. 33 (1904), 559-569.

[7] S. A. R. Robertson, Classifying triangles and quadrilaterals, Math. Gaz. 61 (1977), 3849.

V. Volenec

Department of Mathematics

University of Zagreb

Bijenička cesta 30, HR-10 000 Zagreb

Croatia

E-mail: volenec@math.hr 
Z. Kolar-Begović

Department of Mathematics

University of Osijek

Trg Ljudevita Gaja 6, HR-31 000 Osijek

Croatia

E-mail: zkolar@mathos.hr

R. Kolar-Šuper

Faculty of Education

University of Osijek

Cara Hadrijana 10, HR-31 000 Osijek

Croatia

E-mail: rkolar@foozos.hr

Received: 19.2.2019. 